\title{
Perfil antropométrico e fisiológico de atletas brasileiros de "rugby"
}

CDD. 20.ed. 796.022

796.033

796.333

\author{
André Luiz LOPES* \\ Ricardo Tannhauser SANT'ANA* \\ Bruno Manfredini BARONI* \\ Giovani dos Santos CUNHA ${ }^{* *}$ \\ Regis RADAELLI* \\ Álvaro Reischak de OLIVEIRA* \\ Flávio de Souza CASTRO*
}

*Escola de Educação

Física, Universidade

Federal do Rio Grande do Sul.

**Faculdade de Educação Física, Sociedade de Ginástica Porto Alegre.

\section{Resumo}

0 "Rugby" é um esporte bastante popular internacionalmente e em franca ascensão no Brasil. É caracterizado pela existência de duas posições táticas básicas ("forwards" e "backs"), cujos atletas apresentam demandas funcionais e características físicas distintas. Embora a literatura internacional apresente um número interessante de referências acerca do perfil antropométrico e fisiológico destes atletas, pouca atenção tem sido despendida aos atletas brasileiros. Assim, este trabalho teve como objetivo verificar o desempenho de 20 jogadores amadores de "Rugby" submetidos à ergoespirometria, teste de Wingate e Dinamometria isocinética bem como a comparação entre "backs" $(n=10)$ e "forwards" $(n=10)$ para verificar possiveis diferenças entre as posições táticas. Para determinação dos valores de $\mathrm{VO}_{2}$ e VCO foi utilizado um analisador de gases computadorizado (CPX-D; MedGraphics Cardiorespiratory Diagnostic Systems) para variáveis de força um dinamômetro isocinético Cybex Norm (Lumex \&t Co., Ronkonkoma, USA) e para comparações de composição corporal foi aplicada a técnica de cinco componentes da ISAK. A comparação entre os dados foi verificada por meio do teste $t$ de Student para amostras independentes, sendo que para todas as variáveis foi adotado um índice de significância de $p<0,05$. Os nossos resultados mostram uma diferença significativa entre "backs" e "forwards' nas variáveis $\mathrm{VO}_{2 \max }\left(47,8 \pm 4,5\right.$ e 38,8 $\left.\pm 5,5 \mathrm{ml} \mathrm{kg}^{-1} \cdot \mathrm{min}^{-1}\right)$ - $2^{\circ}$ limiar ventilatório $(38,3 \pm 3,0$ e 31,6 $\left.\pm 4,2 \mathrm{ml} . \mathrm{kg}^{-1} \cdot \mathrm{min}^{-1}\right)$ - potência média $\left(7,5 \pm 0,6\right.$ e 6,3 $\left.\pm 1,1 \mathrm{~W}^{\mathrm{kg}} \mathrm{g}^{-1}\right)$ e trabalho total $(225,7 \pm 18,4$ e 187,9 $\pm 31,7$ J.kg-1) - massa corporal $(78,5 \pm 9,5$ e 101,6 $\pm 12,6$ kg) Massa Adiposa $(24,7 \pm 3,2$ e 29,7 $\pm 4,6 \%)$ Massa Muscular $(48,7 \pm 4,2$ e $44,5 \pm 3,4 \%)$ respectivamente $(p<0,05)$. Sendo assim, evidenciamos a existência de diferenças significativas em algumas das variáveis medidas entre atletas de "Rugby' conforme sua função no jogo. Essa evidência mostra que mesmo os atletas sendo de nivel amador, as características fisiológicas, antropométricas e mecânicas são semelhantes quando comparadas aos jogadores de nível profissional.

UnITERMOS: Antropometria; Ergoespirometria; Teste de Wingate; Dinamometria isocinética.

\section{Introdução}

O "Rugby" é um esporte coletivo, jogado em campo gramado de $100 \times 70$ metros por um período de dois tempos de 40 minutos com intervalo de 10 minutos. $\mathrm{O}$ objetivo principal do jogo é vencer a defesa adversária e apoiar a bola na extremidade final do campo, a qual é chamada de "in-goal', marcando o "try", que tem valor de cinco pontos. Para tal, cada equipe é composta por 15 atletas que são divididos em "backs"(sete jogadores) e "forwards" (oito jogadores), de acordo com a função tática que exercem durante a partida (MCLEAN, 1992).
O "Rugby" é um esporte que exige uma variedade de respostas fisiológicas de seus jogadores, pois o jogo é marcado por repetitivas corridas de alta intensidade e contato corporal (Scott, Roe, CoATs \& Piepoli, 2003 citados por Perella, Noriyuki \& Rossi, 2005). A literatura nos mostra que os "forwards" necessitam de potência física, os "backs" de velocidade e agilidade (NICHOLAS, 1997; SCOTT et al., 2003). Os "backs" são atletas caracterizados por possuírem uma capacidade aeróbia bem desenvolvida e baixo percentual de 
gordura corporal, enquanto que os "forwards" são caracterizados por uma capacidade de produção de força acentuada, percentual de gordura alto e massa muscular bem desenvolvida (DACRES-MANNINGS, ROCHESTER \& FraIL, 2001).

Diversos estudos foram publicados com dados referentes à antropometria e fisiologia de jogadores de "Rugby" em diversos países do mundo (BAKER, 2002; Carlson, Carter, Patterson, Petti, Orfanos, \& Noffal, 1994; Dacres-Mannings, Rochester \&

\section{Métodos}

\section{Amostra}

Foram avaliados 20 jogadores (10 "backs" e 10 "forwards") amadores de "Rugby" da equipe vicecampeã do estado do Rio Grande do Sul de 2009, com idade entre 20 e 40 anos. Perdas amostrais ocorreram durante o procedimento de coleta de dados, de modo a impossibilitar que fossem apresentados os resultados de todos os 20 atletas em todas as avaliaçōes realizadas. $\mathrm{O}$ presente estudo foi aprovado pelo Comitê de Ética em Pesquisa da Universidade Federal do Rio Grande do Sul (protocolo 2008158) e todos os sujeitos assinaram um termo de consentimento livre e esclarecido e estavam cientes do objetivo do presente estudo.

\section{Procedimentos}

Todo o procedimento de coleta de dados foi realizado no Laboratório de Pesquisa do Exercício (LAPEX) da Escola de Educação Física (ESEF) da Universidade Federal do Rio Grande do Sul (UFRGS). Cada atleta realizou duas visitas ao laboratório, intervaladas por um período mínimo de 72 horas. Na primeira sessão, os voluntários foram submetidos à avaliação antropométrica e avaliação da capacidade aeróbia. Na segunda sessão, os mesmos foram submetidos à avaliação da capacidade de produção de força de membros inferiores e avaliação da capacidade anaeróbia. A descrição de cada um destes procedimentos é fornecida a seguir.

\section{Avaliação antropométrica}

As dobras cutâneas foram medidas utilizando um plicômetro (Modelo Harpenden Científico, Marca Cescorf, Porto Alegre, Brasil), diâmetros ósseos por paquímetro e antropômetro (Cescorf, Porto Alegre,
Frail, 2001; Nicholas, 1997; SCOTt et al., 2003; Tong, Bell, Ball \& Winter, 2001). Entretanto, o "Rugby" brasileiro é um esporte em desenvolvimento, com poucas equipes de competição, o que também se reflete na área científica onde não foram encontrados estudos que fizessem referencia a caracterização de atletas dessa modalidade esportiva. Sendo assim, este trabalho teve como objetivo verificar características fisiológicas e de composição corporal de jogadores adultos de "Rugby" de nível amador do Brasil.

Brasil), perímetros usando fita métrica (Sanny, São Bernardo do Campo, São Paulo), massa e estatura medidas por meio de balança e estadiometro (Tânita, Pinheiros, São Paulo). As marcações dos locais e a técnica de tomada das dobras cutâneas seguiram os padrôes da Sociedade Internacional para o Avanço da Cineantropometria (ISAK). Os cálculos da composição corporal foram realizados usando a metodologia de cinco componentes (MARFELl-Jones, OldS, STEWART \& CARTER, 2006 - ISAK). Nessa metodologia são verificadas as medidas de 39 pontos de referência, sendo utilizados para esse trabalho as seguintes variáveis: 1 ) massa corporal; 2) estatura; 3) dobras cutâneas (tríceps, subescapular, bíceps, crista ilíaca, supra espinhal, abdominal, coxa medial e panturrilha); 4) perímetros (cabeça, braço, tórax, cintura, coxa máxima, coxa média, panturrilha, quadril, antebraço e tornozelo);5) diâmetros ósseos (biacromial, tórax transverso, tórax antero-posterior, bi-iliocristal, bi-epicondilar do úmero, bi-estilóide de punho, mão, bi-condilar do fêmur, bi-maleolar); e comprimentos (acrômio-radial, radialestilóide, estilóidea média-dactilóidea, ílio-espinhal banco, trocantérica banco, trocanter-tibial lateral, tibial lateral banco, tibial medial-maleolar medial, comprimento do pé e altura sentado).

\section{Avaliação da capacidade aeróbia (ergoespirometria)}

Foi realizado um protocolo de teste incremental máximo em esteira ergométrica, iniciado com três minutos de corrida a $7 \mathrm{~km} . \mathrm{h}^{-1}$ e seguido de incrementos de $0,5 \mathrm{~km} \cdot \mathrm{h}^{-1}$ a cada $30 \mathrm{~s}$ até a exaustão com inclinação da esteira mantida constante em $1 \%$. Simultaneamente ao teste de esforço máximo foi realizada uma análise das trocas respiratórias através de um equipamento de ergoespirometria (MedGraphics Cardiorespiratory 
Diagnostic Systems, modelo CPX-D, Saint Paul, Minnesota, USA). Os critérios utilizados para verificar se o teste realmente foi máximo foram: a exaustão do atleta; o $\mathrm{R}$ (quociente respiratório) $\geq 1,15$; a $\mathrm{FC}_{\mathrm{m}}$ (frequência cardíaca máxima) do teste $\geq 95 \%$ da FC predita (220-idade); e/ou presença de platô no $\mathrm{VO}_{2}$ mesmo com o incremento da carga. A calibração do volume no pneumotacógrafo foi realizada eletronicamente pelo sistema para a calibração do volume zero. Logo após, foi realizada a calibração do volume com cinco injeções e ejeções de ar em diferentes velocidades através do pneumotacógrafo com uma seringa de três litros. A calibração do analisador de gases foi realizada através do ajuste das concentrações de $\mathrm{O}_{2}$ e $\mathrm{CO}_{2}$ de acordo com as concentrações dos cilindros de referência $\left(21 \%\right.$ de $\mathrm{O}_{2}$ e nitrogênio para balanço) e de calibração $\left(12 \% \mathrm{O}_{2}\right.$ e $6 \% \mathrm{CO}_{2}$ e nitrogênio para balanço). Toda a análise para a determinação do $\mathrm{VO}_{2 \max }$ foi realizada pelo método "breath by breath" (Dos Santos Cunha, Garcia Célia, Ribeiro \& Reischak de Oliveira, 2008).

\section{Avaliação da capacidade anaeróbia (teste de Wingate)}

Para determinação da capacidade anaeróbia foi realizado o teste de Wingate em um cicloergômetro Cybex (Lumex \& Co., Ronkonkoma, USA). Antes dos testes, os atletas realizaram um período de cinco minutos de aquecimento no cicloergômetro com rotação superior a $60 \mathrm{rpm}$. Após serem instruídos e terem todas as possíveis dúvidas sanadas pelos pesquisadores, os atletas realizaram o teste de capacidade anaeróbia conforme as recomendações propostas por INBAR, BAR-OR e SKINNER (1996): 30 segundos de exercício máximo em cicloergômetro com uma carga correspondente a $7,5 \%$ da massa corporal, objetivando atingir a máxima rotação desde o início do teste. Ressalta-se que estímulo verbal foi fornecido pelos avaliadores de maneira padronizada e constante durante todos os testes.

\section{Avaliação da capacidade de produção de força de membros inferiores (dinamometria isocinética)}

A capacidade máxima de produção de torque isométrico de extensores do joelho foi determinada por meio de três contrações voluntárias isométricas máximas (CVIMs) realizadas no ângulo de $60^{\circ}$ de flexão de joelho $\left(0^{\circ}=\right.$ extensão total de joelho) em um dinamômetro isocinético Cybex Norm (Lumex \& Co., Ronkonkoma, USA), o qual foi calibrado de acordo com as instruçōes do fabricante. Todas as CVIMs tiveram duração de cinco segundos, sendo respeitado intervalo de dois minutos para recuperação entre as contraçōes para minimizar possíveis efeitos da fadiga. Após, a capacidade máxima de geração de torque concêntrico de extensores do joelho foi determinada por meio de três contrações voluntárias dinâmicas máximas (CVDMs) realizadas na velocidade angular de $60 \% \mathrm{~s}$ e amplitude total de movimento dos avaliados, também com intervalo de dois minutos entre as contrações para evitar efeitos da fadiga muscular. Os sujeitos foram instruídos a realizar o máximo esforço em cada contração realizada (tanto nas CVIMs quanto nas CVDMs), além de estimulados verbalmente de forma padronizada durante todas as contraçôes. Os mais altos valores de torque, respectivamente verificados nas CVIMs e CVDMs, foram considerados para posterior análise.

\section{Análise estatística}

As variáveis antropométricas consideradas para análise estatística foram: 1) massa corporal; 2) estatura; 3) massa adiposa; 4) massa muscular; 5) massa óssea; 6) massa residual; e 7) massa epitelial.

A capacidade aeróbia foi avaliada pelos valores de: 1) consumo máximo de oxigênio $\left.\left(\mathrm{VO}_{2 \max }\right) ; 2\right)$ frequência cardíaca máxima atingida no teste ergoespirométrico $\left.\left(\mathrm{FC}_{\max }\right) ; 3\right)$ segundo limiar ventilatório $\left.\left(\mathrm{LV}_{2}\right) ; 4\right)$ percentual do $\mathrm{VO}_{2 \max }$ em que foi atingido o $\mathrm{LV}_{2}\left(\% \mathrm{VO}_{2 \max }\right.$ no LV2); 5) frequência cardíaca em que foi atingido o $\mathrm{LV}_{2}$; e 6) percentual da FCmax em que foi atingido o $\mathrm{LV}_{2}$.

A capacidade anaeróbia foi avaliada por meio dos valores de: 1) potência máxima, que consiste no máximo valor de potência obtido durante o teste; 2) potência média, obtido pelo cálculo da média de todos os valores de potência obtidos durante o teste; e 3) trabalho total realizado durante o teste.

Por fim, a análise da capacidade de produção de força de membros inferiores envolveu os valores de pico de torque obtidos respectivamente durante as CVIMs e CVDMs.

Foi realizada a análise descritiva dos resultados, sendo apresentados os valores de média e desvio padrão dos "backs" e "forwards" para todas as variáveis avaliadas. A normalidade da distribuição foi verificada através do "z-score ", sendo adotado como critério de exclusão indivíduos que apresentassem valores iguais ou superiores a 2,00 desvios padrão. A comparação entre "backs" e "forwards" foi realizada por meio do teste $t$ de Student para amostras independentes, sendo que para todas as variáveis foi adotado um índice de significância de p $<0,05$. 


\section{Resultados}

A análise dos resultados das avaliações antropométricas revela que os "forwards" apresentam massa corporal total e percentual de massa adiposa significativamente superior aos "backs", que por sua vez apresentam maior percentual de massa muscular (TABELA 1).

Em relação à capacidade aeróbia, as avaliações ergoespirométricas demonstram que os "backs" apresentam níveis superiores de $\mathrm{VO}_{2 \max }$ e $\mathrm{LV}_{2}$ (em valores normalizados pela massa corporal dos atletas), conforme apresentado na TABELA 2. Da mesma forma, os "backs" demonstram superioridade na execução do teste de Wingate através de valores de potência média e trabalho total (também em valores normalizados pela massa corporal total), apresentados na TABELA 2.

TABELA 1- Valores relativos às características antropométricas dos atletas (9 "backs"; 10 "forwards").

* TABELAS 1 e 2 Diferença significativa entre "backs" e "forwards" $p<0,05$.

\begin{tabular}{lccc}
\hline & Backs & Forwards & Valor de p \\
\hline Massa corporal (kg) * & $78,5 \pm 9,5$ & $101,6 \pm 12,6$ & 0,033 \\
Estatura (cm) & $175,1 \pm 5,7$ & $179,2 \pm 5,5$ & 0,788 \\
Massa Adiposa (\%) * & $24,7 \pm 3,2$ & $29,7 \pm 4,6$ & 0,039 \\
Massa Muscular (\%) * & $48,7 \pm 4,2$ & $44,5 \pm 3,4$ & 0,041 \\
Massa Óssea (\%) & $10,1 \pm 1,6$ & $9,6 \pm 0,8$ & 0,234 \\
Massa Residual (\%) & $11,4 \pm 0,7$ & $11,9 \pm 1,3$ & 0,845 \\
Massa Epitelial (\%) & $4,8 \pm 0,4$ & $4,1 \pm 0,5$ & 0,775 \\
\hline
\end{tabular}

TABELA 2 - Valores relativos a capacidade aeróbia (ergoespirometria: 10 "backs"; 10 "forwards") e anaeróbia (teste de Wingate: 7 "backs"; 10 "forwards") dos atletas.

\begin{tabular}{|c|c|c|c|c|}
\hline & & Backs & Forwards & Valor de $p$ \\
\hline \multirow{6}{*}{ 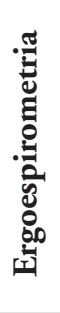 } & $\mathrm{VO}_{2 \max }\left(\mathrm{ml} \cdot \mathrm{kg}^{-1} \cdot \mathrm{min}^{-1}\right)^{*}$ & $47,8 \pm 4,5^{*}$ & $38,8 \pm 5,5$ & 0,001 \\
\hline & $\mathrm{FC}_{\mathrm{Max}}(\mathrm{bpm})$ & $196,1 \pm 14,9$ & $187,6 \pm 7,6$ & 0,126 \\
\hline & $\mathrm{LV}_{2}\left(\mathrm{ml} \cdot \mathrm{kg}^{-1} \cdot \mathrm{min}^{-1}\right)^{*}$ & $38,3 \pm 3,0^{*}$ & $31,6 \pm 4,2$ & 0,001 \\
\hline & $\% \mathrm{VO}_{2 \max }$ no $\mathrm{LV}_{2}$ & $80,7 \pm 9,8$ & $82,2 \pm 11,5$ & 0,754 \\
\hline & FC no $\mathrm{LV}_{2}(\mathrm{bpm})$ & $184,6 \pm 16,3$ & $179,7 \pm 8,9$ & 0,414 \\
\hline & $\% \mathrm{FC}_{\max }$ no $\mathrm{LV}_{2}$ & $94,1 \pm 2,2$ & $95,8 \pm 2,8$ & 0,146 \\
\hline \multirow{3}{*}{ 莺 } & Potência máxima $\left(\mathrm{W} \cdot \mathrm{kg}^{-1}\right)$ & $9,9 \pm 0,5$ & $9,0 \pm 1,4$ & 0,087 \\
\hline & Potência média (W.kg-1) * & $7,5 \pm 0,6^{*}$ & $6,3 \pm 1,1$ & 0,008 \\
\hline & Trabalho total $\left(\mathrm{J} . \mathrm{kg}^{-1}\right)^{*}$ & $225,7 \pm 18,4^{*}$ & $187,9 \pm 31,7$ & 0,008 \\
\hline
\end{tabular}

A FIGURA 1 complementa as informaçoes acerca da capacidade anaeróbia destes atletas, demonstrando o comportamento de "backs" e "forwards" durante o teste de Wingate.

Por fim, a FIGURA 2 apresenta os resultados relativos à capacidade de produção de força de membros inferiores dos atletas. Os resultados demonstraram que não houve diferença significativa entre os atletas das duas posiçôes em relação aos valores de pico de torque obtidos durante as CVIMs e CVDMs de extensão de joelho. 


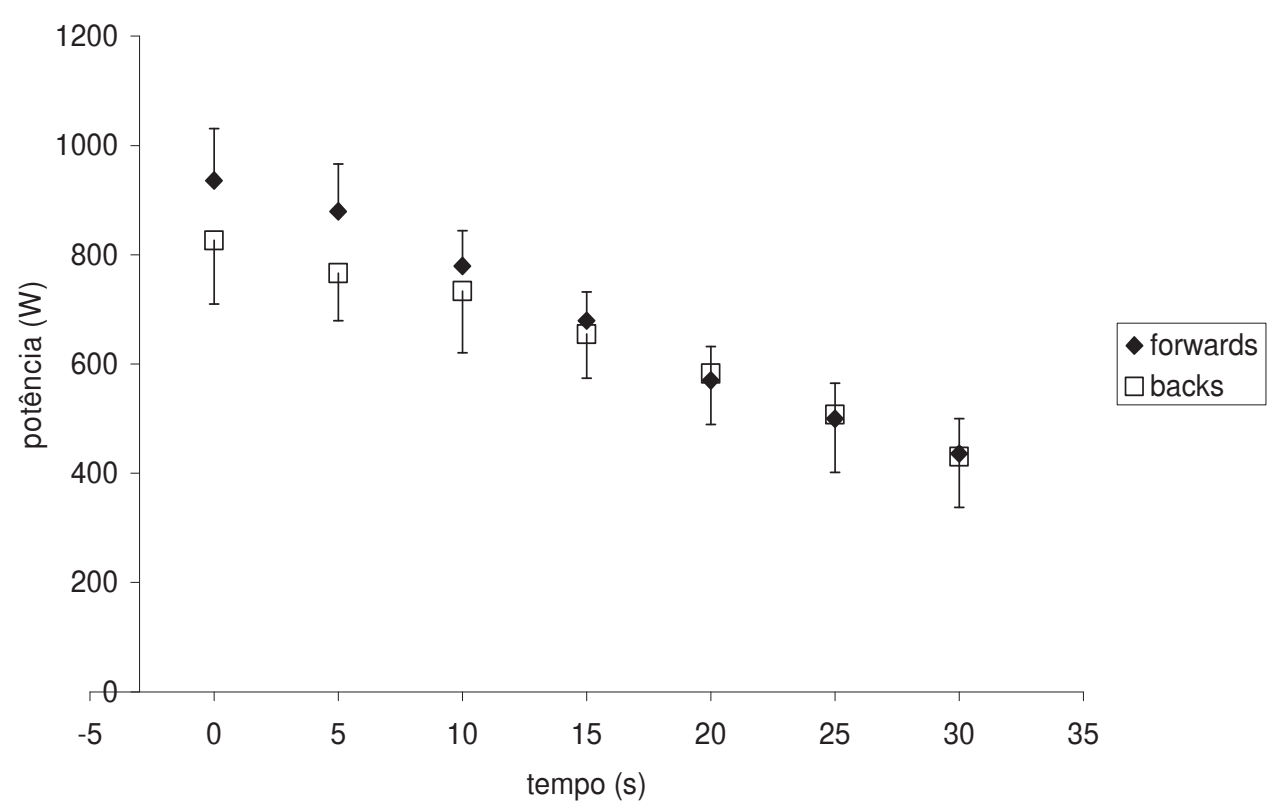

FIGURA 1 - Plotagem ilustrativa representando os valores absolutos de potência obtidos no teste de Wingate (7 "backs"; 10 "forwards").

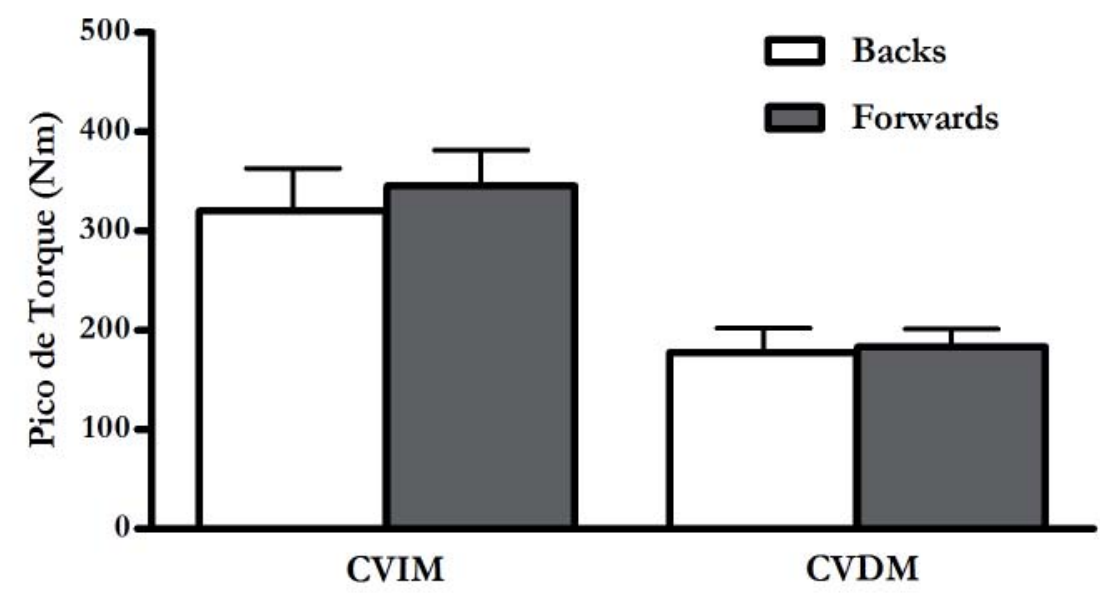

FIGURA 2 - Pico de torque de extensores do joelho em contrações voluntárias isométricas máximas (CVIM) e contrações voluntárias dinâmicas máximas (CVDM) (8 "backs”; 9 "forwards”).

\section{Discussão}

O "Rugby" é um esporte que cresce em popularidade no mundo todo, porém ainda há um número restrito de estudos sobre as características antropométricas e fisiológicas de seus jogadores bem como sobre as demandas metabólicas do jogo (NiCHOLAS, 1997). Os estudos encontrados são direcionados a verificação destes aspectos em jogadores de ligas profissionais, localizadas em países da Europa, África do Sul, Nova Zelândia e Austrália (DACres-Mannings, Rochester \& Frail, 2001; MeIR, NeWton, Curtis,
FArdell \& Butler, 2001; Quarried, HaNdCOCKT, Wallert, Chalmers, ToOmeYl \& Wilsont, 1995). Não foram encontrados trabalhos que avaliem e caracterizem jogadores de "Rugby" brasileiros. Assim, este trabalho pode ser considerado pioneiro na caracterização destes atletas de nível amador do ponto de vista antropométrico e fisiológico.

Nossos resultados denotam a existência de diferenças significativas entre as características antropométricas dos atletas das duas posições táticas. Enquanto os 
"forwards" apresentaram uma maior massa corporal total e um maior percentual de massa adiposa, os "backs" apresentaram um maior percentual de massa muscular. Em parte, os resultados podem ser explicados pela função desempenhada durante o jogo, onde os "forwards" passam mais tempo em embate físico com os oponentes, sendo interessante possuírem massa corporal avantajada. Porém, é importante salientar que essa superioridade em relação à quantidade de massa corporal total é acompanhada por um maior percentual de massa adiposa, ou seja, tecido não contrátil que aumenta o volume corporal sem exercer função sobre a capacidade de produção de força dos atletas.

Por sua vez, os "backs" estão envolvidos em situações que necessitam velocidade e agilidade (Nicholas, 1997; ScotT et al., 2003) e passam mais tempo em corridas livres (DuThie, Pyne \& HoOPER, 2003), permitindo a este grupo cobrir maiores distâncias dentro do campo de jogo, o que ajuda a explicar o menor percentual de gordura encontrado neste grupo (QUARRIED et al., 1995). Os valores de composição corporal, significativamente diferentes entre "backs" e "forwards", concordam com estudos prévios envolvendo atletas da modalidade em questão (BAKer, 2002; Carlson et al., 1994; DacresMannings, Rochester \& Frail, 2001; Nicholas, 1997; Tong et al., 2001).

Nossos dados também demonstram os "backs" apresentaram um $\mathrm{VO}_{\text {2máx }}$ significativamente superior aos "forwards", o que também pode estar relacionado ao maior envolvimento dos primeiros em situaçôes de corridas livres (DuthiE, Pyne \& HoOper, 2003), enquanto que os "forwards" estão mais envolvidos em situações de contato e força (NICHOLAS, 1997; SCOTT et al., 2003). Tais resultados também vão ao encontro dos achados de estudos internacionais que avaliaram atletas profissionais de "Rugby" (Bell, 1995; Brooks, Fuller, Kemp \& REDDIN, 2005; QuARRIED et al., 1995).

Os valores reportados internacionalmente em atletas profissionais de "Rugby" variam entre 48,6 e $62,6 \mathrm{ml} \cdot \mathrm{kg}^{-1} \cdot \mathrm{min}^{-1}$, mostrando considerável variação nesta população, o que demonstra características específicas de cada jogador conforme a função no jogo (O'Connor, 1995). Alguns autores têm comparado valores de $\mathrm{VO}_{2 \text { máx }}$ de jogadores de "Rugby" com o de atletas de futebol e "ice hockey", encontrando os respectivos valores de $51,1 \pm 1,4 \mathrm{ml} \cdot \mathrm{kg}^{-1} \cdot \mathrm{min}^{-1}, 57,8$ \pm 6,5 ml. $\mathrm{kg}^{-1} \cdot \mathrm{min}^{-1}$ e $61,8 \pm 1,8 \mathrm{ml} \cdot \mathrm{kg}^{-1} \cdot \mathrm{min}^{-1}$ (BOYLE, Mahoney \& Wallace, 1994; Warrington, Ryan, Murray, Duffy \& KirWan, 2001; Williams, Reid \& CoutTs 1973). Além disso, estudos que avaliaram jogadores amadores de "Rugby" mostraram que estes apresentam valores de $\mathrm{VO}_{2 \text { máx }} 20$ a 42\% menores a jogadores profissionais (CUNNIFFE, PROCTOR, BAKER \& DAVIES, 2009; GABBETT, 2000), podendo ser facilmente explicado pelo menor volume e intensidade de treinamento por parte dos atletas amadores. De fato, a carga horária de treinamento dos jogadores amadores fora reportada como sendo entre $30 \%$ a $53 \%$ menor quando comparado aos jogadores profissionais, que possuíam cinco a 7,5 horas de treinamento por semana (GabBett, 2000; Phillips, Standen, \& BATt, 1998; Stephenson, Gissane, \& Jennings, 1996).

ScOTT et al. (2003) avaliaram o $\mathrm{VO}_{2 \text { pico }}$ de 28 jogadores profissionais, 15 "backs" e 13 "forwards". Os resultados mostraram uma superioridade de $\mathrm{VO}_{2 \text { máx }}$ em favor dos "backs" $\left(48,3 \pm 2,1 \mathrm{ml} \cdot \mathrm{kg}^{-1} \cdot \mathrm{min}^{-1}\right) \mathrm{em}$ comparação aos "forwards" (41,2 $\left.\pm 2,7 \mathrm{ml} \cdot \mathrm{kg}^{-1} \cdot \mathrm{min}^{-1}\right)$. Entretanto, os "forwards" apresentaram maior massa corporal e maior estatura ("forwards": $104 \pm 2,1 \mathrm{~kg}$ e 190,2 $\pm 2,2$ cm; "backs": $86,3 \pm 1,7$ kg e 179,5 \pm $1,3 \mathrm{~cm})$. Estes valores de $\mathrm{VO}_{2 \text { máx }}$ e massa corporal parecem se aproximar dos valores observados no presente estudo, enquanto a estatura dos atletas profissionais parece ser superior aos dos atletas amadores avaliados neste trabalho.

Nossos resultados demonstram que o $2^{\circ}$ limiar ventilatório corresponde a 38,3 $\pm 3,0 \mathrm{ml} \cdot \mathrm{kg}^{-1} \mathrm{~min}^{-1} \mathrm{e}$ $31,6 \pm 4,2$ ml.kg-1 min-1 para "backs" e "forwards", respectivamente dos valores máximos de consumo de oxigênio. ScotT et al. (2003) encontraram valores de limiar anaeróbio inferiores ao nosso, que correspondiam a 17,7 $\pm 1,3 \mathrm{ml} . \mathrm{kg}^{-1} \mathrm{~min}^{-1}$ e $18,6 \pm 2 \mathrm{ml} . \mathrm{kg}^{-1} \mathrm{~min}^{-1}$ para "backs" e "forwards", respectivamente. Tem sido sugerido que uma elevada capacidade aeróbia melhora a recuperação entre os exercícios intermitentes de alta intensidade, provavelmente por remover mais rapidamente o lactato sanguíneo e restaurar os níveis de ATP-PCr. Este fato demonstra a importância de um atleta possuir um elevado limiar anaeróbio. Provavelmente, atletas que possuem um elevado limiar anaeróbio conseguem suportar exercícios de alta intensidade com uma menor parcela de energia proveniente da via glicolítica anaeróbia, assim diminuindo as concentrações de lactato e $\mathrm{H}+$, resultando em uma melhor manutenção do desempenho físico.

Os resultados de capacidade anaeróbia expressos em valores absolutos (FIGURA 1) mostram diferença entre os grupos em relação à potência desenvolvida nos dois primeiros momentos analisados, o que transmite a idéia inicial de haver superioridade dos "forwards" nesta variável. Entretanto, conforme mencionado pelos próprios criadores do teste de 
Wingate, ao se comparar indivíduos com massa corporais diferentes é importante que seja realizada uma normalização dos valores obtidos pela massa corporal de cada avaliado (INBAR, BAR-OR \& SKINNER, 1996). Além disso, tendo em vista que o "Rugby" é uma modalidade em que o atleta realiza transporte constante da própria massa corporal para execução das açôes do jogo, a maneira mais adequada para comparar a capacidade anaeróbia de "backs" e "forwards" é através de dados normalizados pela massa corporal total, como expresso na TABELA 2. Assim, nossos resultados demonstram que os backs apresentaram superioridade em relação à capacidade anaeróbia, expressa por maiores valores de potência média e trabalho total, além de uma tendência em atingir valores superiores de potência máxima. Mesmo assim, optou-se pela apresentação da FIGURA 1 com finalidade de demonstração do comportamento de "backs" e "forwards" durante o teste de Wingate.

Diante das demandas do esporte e superioridade de massa corporal total apresentada pelos "forwards", esperávamos que os atletas desta posição atingissem valores superiores de geração de torque de extensores de joelho, entretanto, nossos resultados refutam nossa hipótese inicial. Mesmo no presente estudo, tendo sido encontrada diferença entre os dois grupos na potência média, e essa apresentando forte relação com a força máxima (BAKER \& NANCE, 1999), não foi observado diferença entre "backs" e "fowards" nos valores de CVIM e CVDM (FIGURA 2). Nossos resultados corroboram com os de MEIR et al. (2001) que também não observaram diferença entre valores de produção de força de membros inferiores entre "backs" e "fowards". Além disso, os resultados do presente estudo podem sugerir que a massa corporal pode não ser uma das melhores preditoras da capacidade de produção de força em atletas de "Rugby". Como observado por BAKER (2002), que após a avaliação da capacidade de produção de força e da massa corporal de atletas de "Rugby" de diferentes níveis, não há linearidade no aumento da capacidade de produção de força e no aumento de massa corporal dos sujeitos.

\title{
Conclusão
}

Concluímos que existem diferenças significativas entre jogadores amadores de "Rugby" que atuam como "backs" e "forwards" no que diz respeito à composição corporal (massa corporal total, massa adiposa e massa muscular), capacidade aeróbia $\left(\mathrm{VO}_{2 \text { máx }}\right.$ e $\left.\mathrm{LV}_{2}\right)$ e capacidade anaeróbia (potência média e trabalho total). Assim, as diferenças observadas nas características antropométricas e fisiológicas de "backs" e "forwards" demonstram a necessidade da seleção de sujeitos com diferentes componentes físicos para se obter a formação de uma equipe de competição de "Rugby".
Tendo em vista o número restrito de participantes avaliados no presente estudo, conclusóes contundentes acerca dos parâmetros avaliados tornam-se limitadas. No entanto, tendo em vista a lacuna observada na literatura nacional a respeito do assunto, acreditamos que o pioneirismo deste trabalho e a qualidade dos métodos de avaliação utilizados no presente estudo contribuam com a prática profissional de treinadores e preparadores físicos, além de incentivar o desenvolvimento de novos estudos com esta crescente população.

\begin{abstract}
Anthropometric and physiological profile of Brazilian rugby athletes

Rugby is a very popular sport around the world and on the rise in Brazil. It is characterized by the existence of two basic tactics positions (forwards and backs), in which athletes have distinguishing functional demands and physical characteristics. Although the international literature presents a number of interesting data about the physiological and anthropometric profile of these athletes, little attention has been spent to Brazilian athletes. This study aimed to verify the performance of 20 amateur rugby players and to compare the aerobic power $\left(\mathrm{VO}_{2 \max }\right)$, anaerobic endurance and isokinetic strength in backs $(n=10)$ and forwards $(n=10)$. A computerized gas analyzer (CPX-D - MGC) was used to determine $\mathrm{VO}_{2}$ and $\mathrm{VCO}_{2}$. A Wingate test was used to determine the anaerobic endurance. An isokinetic dynamometer
\end{abstract}


(Cybex Norm) was used to measure isokinetic strength. The body composition was evaluated according to five components of ISAK. The data were compared through Student $t$ test for independent samples ( $p$ $<0.05$ ). Our results show a significant difference between backs and forwards in the variables V02max $\left(47.8 \pm 4.5\right.$ and $\left.38.8 \pm 5.5 \mathrm{ml} . \mathrm{kg}^{-1} \cdot \mathrm{min}^{-1}\right) ; 2$ nd ventilatory threshold $\left(38.3 \pm 3.0\right.$ and $31.6 \pm 4.2 \mathrm{ml}^{\mathrm{kg}} \mathrm{kg}^{-1}$.

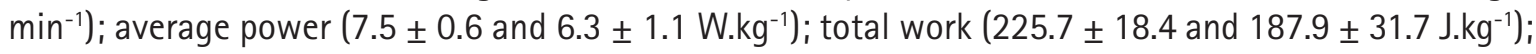
body weight $(78.5 \pm 9.5$ and $101.6 \pm 12.6 \mathrm{~kg})$; fat mass $(24.7 \pm 3,2$ and $29.7 \pm 4.6 \%)$ and muscle mass $(48.7 \pm 4.2$ and $44.5 \pm 3.4 \%)$ respectively $(p<0.05)$. Thus, there are significant differences, related to the tactic position and function, in Rugby athletes. Furthermore our results shows that even at the amateur level athletes, physiological and anthropometric characteristics are similar when compared to professional level players.

UNITERMS: Anthropometry; Aerobic power; Wingate test; Isokinetic dynamometry.

\section{Referências}

BAKER, D. Differences in strength and power among junior- high, senior-high, college-aged, and elite professional rugby league players. The Journal of Strength and Conditioning, Champaign, v.16, n.4, p.581-85, 2002.

BAKER, D.; NANCE, S. The relation between strength and power in professional rugby league players. The Journal of Strength and Conditioning Research, Champaign, v.13, n.3, p.224-29, 1999.

BELL, W. The estimation of body density in rugby union football players. British Journal of Sports Medicine, London, v.29, n.1, p.46-51, 1995.

BOYLE, P.M.; MAHONEY, C.A.; WALLACE, W.F. The competitive demands of elite male field hockey. Journal of Sports Medicine Physical Fitness, Turin, v.34, n.3, p.235-41, 1994.

BROOKS, J.H.; FULLER, C.W.; KEMP, S.P.; REDDIN, D.B. A prospective study of injuries and training amongst the England 2003 Rugby World Cup squad. British Journal of Sports Medicine, London, v.39, n.5, p.288-93, 2005.

CARLSON, B.R.; CARTER, J.E.; PATTERSON, P.; PETTI, K.; ORFANOS, S.M.; NOFFAL, G.F. Physique and motor performance characteristics of US national rugby players. Journal of Sports Sciences, London, v.12, n.4, p.403-12, 1994. CUNNIFFE, B.; PROCTOR, W.; BAKER, J.S.; DAVIES, B. An evaluation of the physiological demands of elite rugby union using Global Position System tracking software. The Journal of Strength and Conditioning Research, Champaign, v.23, n.4, p.1195-203, 2009.

DACRES-MANNINGS, S.S.; ROCHESTER, S.; FRAIL, H. Anthropometric profiles of Australian Rugby Institute, Club and State Level Rugby Union Players, 2001. Acesso em: 07 out. 2009.

DOS SANTOS CUNHA, G.; GARCIA CÉLIA, F.; RIBEIRO, J.L.; REISCHAK DE OLIVEIRA, A. Effects of the biological maturation on maximal oxygen uptake and ventilatory breakpoint of Brazilian soccer players. Gazzetta Medica Italiana Archivio per Le Scienze Mediche, Torino, v.167, n.2, p.43-9, 2008.

DUTHIE, G.; PYNE, D.; HOOPER, S. Applied physiology and game analysis of rugby union. Sports Medicine, Ackland, v.33, n.13, p.973-91, 2003.

GABBETT, T. J. Physiological and antropometric characteristics of amateur rugby league players. British Journal of Sports Medicine, London, v.34, n.4, p.303-7, 2000.

INBAR, O.; BAR-OR, O.; SKINNER, J.S. The Wingate anaerobic test. Champaign: Human Kinetics, 1996. MARFELL-JONES, M.; OLDS, T.; STEWART, A.; CARTER, J.E.L. (Eds.). International Standards for Anthropometric Assessment. Potchefstroom: North-West University, 2006.

McLEAN, D.A. Analysis of the physical demands of international rugby union. Journal of Sports Sciences, London, v.10, n.3, p.285-96, 1992.

MEIR, R.; NEWTON, R.; CURTIS, E.; FARDELL, M.; BUTLER, B. Physical fitness qualities of professional rugby league football players: determination of positional differences. The Journal of Strength and Conditioning Research, Champaign, v.15, n.4, p.450-8, 2001.

NICHOLAS, C.W. Antropometric and physhiological characteristics of rugby union football players. Sports Medicine, Ackland, v.23, n.6, p.375-96, 1997.

O'CONNOR, D. Fitness profile rugby league of professional players. Journal of Sports Sciences, London, v.13, p.505, 1995. 
PERRELLA, M.M.; NORIYUKI, P.S; ROSSI, L. Avaliação da perda hídrica durante treino intenso de rugby. Revista Brasileira de Medicina do Esporte, São Paulo, v.11, n.4, p 229-32, 2005.

PHILLIPS, L.H.; STANDEN, P.J.; BATT, M.E. Effects of seasonal change in rugby league on the incidence of injury. British Journal of Sports Medicine, London, v.32, n.2, p.144-48, 1998.

QUARRIED, K.L.; HANDCOCKT, P.; WALLERT, A.E.; CHALMERS, D.J.; TOOMEYL, M.J.; WILSONT, B.D. The New Zealand rugby injury and performance project. III. Anthropometric and physical performance characteristics of players. British Journal of Sports Medicine, London, v.29, n.4, p.263-70, 1995.

SCOTT, A.C.; ROE, N.; COATS, A.J.; PIEPOLI, M.F. Aerobic exercise physiology in a professional Rugby Union Team. International Journal of Cardiology, Anglia, v.87, n.2-3, p.173-7, 2003.

STEPHENSON, S.; GISSANE, C.; JENNINGS, D. Injury in rugby league: a four year prospective survey. British Journal of Sports Medicine, London, v.30, n.4, p.331-4, 1996.

TAKARADA, Y. Evaluation of muscle damage after a rugby match with special reference to tackle plays. British Journal of Sports Medicine, London, v.37, n.5, p.416-9, 2003.

TONG, R.J.; BELL, W.; BALL, G.; WINTER, E.M. Reliability of power output measurements during repeated treadmill sprinting in rugby players. Journal of Sports Sciences, London, v.19, n.4, p.289-97, 2001.

WARRINGTON, G.; RYAN, C.; MURRAY, F.; DUFFY, P.; KIRWAN, J.P. Physiological and metabolic characteristics of elite tug of war athletes. British Journal of Sports Medicine, London, v.35, n.6, p.396-401, 2001.

WILLIAMS, C.; REID, R.M.; COUTTS, R. Observations on the aerobic power of university rugby players and professional soccer players. British Journal of Sports Medicine, London, v.7, p.390-1, 1973.

\begin{tabular}{r|r} 
ENDEREÇo & \\
André Luiz Lopes & \\
Laboratório de Pesquisa do Exerć́cio & \\
Escola de Educação Física & Recebido para publicação: 13/ 10/2010 \\
1a. Revisão: 16/05/2011 \\
Rniversidade Federal do Rio Grande do Sul & 2a. Revisão: 16/06/2011 \\
90690-200 - Porto Alegre - RS - BRASIL & Aceito: 13/ 07/ 2011 \\
e-mail: andregym23@hotmail.com &
\end{tabular}

